\title{
Synthesis of Pentalene Systems Employing a Sequence of Pauson-Khand Reaction, Michael Reaction, and Desilylation
}

Armand Becheanu, Thomas Bell, Sabine Laschat, Angelika Baro, Wolfgang Frey, Nelli Steinke, and Peter Fischer

Institut für Organische Chemie, Universität Stuttgart, Pfaffenwaldring 55, D-70569 Stuttgart, Germany

Reprint requests to Prof. Dr. S. Laschat. E-mail: sabine.laschat@oc.uni-stuttgart.de

Z. Naturforsch. 61b, 589 - 596 (2006); received January 17, 2006

The utility of cyclopentadiene (4) in intermolecular Pauson-Khand reactions was investigated. Subsequently, a 1,4-addition of lithium organocuprates was carried out followed by desilylation. This synthetic concept allows the preparation of $\beta$-functionalized bicyclo[3.3.0]octane derivatives 1a-c in only three steps in total yields up to $53 \%$.

Key words: Bicyclo[3.3.0]octanes, Cyclopentadiene, Cuprate Addition, Desilylation, Trimethylsilylacetylene

\section{Introduction}

Over the last decade the Pauson-Khand reaction, a cobalt-mediated $[2+2+1]$ cocyclization of an alkyne, an alkene and carbon monoxide to give a cyclopentenone has been extensively investigated and many applications have been reported [1]. Bicyclo[3.3.0]octanes which are key structural motifs in many terpenoid natural products [2], may be accessible directly via PausonKhand reaction of cyclopentadiene (4) with a functionalized alkyne 5 (Scheme 1). The resulting enone 3 might be submitted to conjugate additions and the alkene also can be further manipulated.

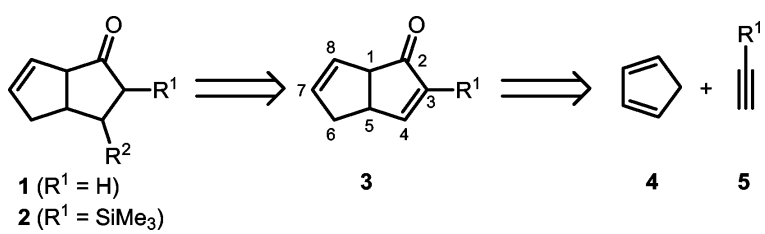

Scheme 1. Retrosynthetic pathway of bicyclo[3.3.0]octane derivatives $\mathbf{1}$ and $\mathbf{2}$.

Surprisingly, little information is available about the use of cyclopentadiene (4) in Pauson-Khand reactions. We anticipated that acetylene or trimethylsilylacetylene should give access to the $\alpha$-unsubstituted ketones 1. In an early paper Pauson reported the preparation of 3-alkyl-substituted pentalenones $3\left(\mathrm{R}^{1}=\right.$ alkyl) in up to $60 \%$ yield from the correspond- ing alkyne dicobalthexacarbonyl complex and cyclopentadiene [3]. In an alternative approach Schore isolated $9 \%$ of bicyclo[3.3.0]octa-3,7-diene-2,6-dione by reacting acetylene with dicobaltoctacarbonyl in benzene [4]. In this paper we wish to disclose our exploration of a synthesis route to target bicyclo[3.3.0]octanes by using cyclopentadiene (4) as starting material. For comparison the corresponding derivatives containing norbornene and norbornadiene instead of cyclopentadiene were investigated as well.

\section{Results and Discussion}

The Pauson-Khand reaction of cyclopentadiene (4) with acetylene gave compound 3 [5] $\left(\mathrm{R}^{1}=\mathrm{H}\right)$ (Scheme 1) in only very low yield $(9 \%)$. As the removal of the trimethylsilyl group in Pauson-Khand cyclization products is well documented in the literature [6], acetylene was replaced with trimethylsilylacetylene (5) as an alternative precursor. Alkyne $\mathbf{5}$ was treated with dicobaltoctacarbonyl in $\mathrm{CH}_{2} \mathrm{Cl}_{2}$ at room temperature $[6 a, b, 7]$ to give the cobalt-alkyne complex 6. According to literature procedures $[6 a, b, 7]$ the latter was reacted first with norbornene (7a) and norbornadiene (7b) in the presence of NMO (Scheme 2). The corresponding TMS-substituted derivatives $8 \mathbf{a}$ and $\mathbf{8 b}[6 a, b, 7,8]$ were isolated in $91 \%$ and $63 \%$ yield, respectively. 


\begin{tabular}{|c|c|c|c|c|c|c|c|}
\hline \multirow[b]{2}{*}{ Entry } & \multicolumn{3}{|c|}{ Starting materials } & \multicolumn{2}{|c|}{ Reaction conditions } & \multicolumn{2}{|c|}{ Products } \\
\hline & Enone & Reagent & Solvent & $\mathrm{T}\left[{ }^{\circ} \mathrm{C}\right]$ & $t[\mathrm{~h}]$ & & Yield [\%] \\
\hline 1 & $\mathbf{8 a}$ & TBAF & THF & 20 & 72 & $9 \mathbf{a}$ & 47 \\
\hline 2 & $\mathbf{8 a}$ & $\mathrm{MeSO}_{3} \mathrm{H}$ & $\mathrm{MeOH}$ & 20 & 16 & $9 \mathbf{a}$ & 40 \\
\hline 3 & $8 \mathbf{a}$ & $\mathrm{CsOH}$ & DMF & -20 & 1 & $9 \mathbf{a}$ & 7 \\
\hline 4 & $\mathbf{8 a}$ & $\mathrm{LiOH}$ & DMF & 20 & 12 & $9 \mathbf{a}$ & - \\
\hline 5 & $\mathbf{8 a}$ & TASF & DMF & 20 & 12 & $9 \mathbf{a}$ & $<1$ \\
\hline 6 & $\mathbf{8 a}$ & $\mathrm{Bu}_{4} \mathrm{NOH}$ & DMF & -20 & 1 & $9 \mathbf{a}$ & 12 \\
\hline 7 & $\mathbf{8 a}$ & $\mathrm{Bu}_{4} \mathrm{NOH}$ & DMF & 0 & 5 & $9 \mathbf{a}$ & 83 \\
\hline 8 & $\mathbf{8 a}$ & $\mathrm{Bu}_{4} \mathrm{NOH}$ & DMF & 20 & 5 & $9 \mathbf{a}$ & 90 \\
\hline 9 & $\mathbf{8 a}$ & $\mathrm{Bu}_{4} \mathrm{NOH}$ & THF & 20 & 12 & $9 \mathbf{a}$ & 98 \\
\hline 10 & $8 b$ & $\mathrm{MeSO}_{3} \mathrm{H}$ & $\mathrm{Et}_{2} \mathrm{O}$ & 20 & 12 & $9 b$ & - \\
\hline 11 & $8 b$ & $\mathrm{Bu}_{4} \mathrm{NOH}$ & DMF & 20 & 12 & $9 b$ & 68 \\
\hline 12 & $8 b$ & $\mathrm{Bu}_{4} \mathrm{NOH}$ & $\mathrm{Et}_{2} \mathrm{O}$ & 20 & 12 & $9 b$ & 83 \\
\hline 13 & $3 \mathbf{a}$ & $\mathrm{MeSO}_{3} \mathrm{H}$ & $\mathrm{Et}_{2} \mathrm{O}$ & 20 & 5 & $3 b$ & - \\
\hline 14 & $3 \mathbf{a}$ & $\mathrm{Bu}_{4} \mathrm{NOH}$ & DMF & 20 & 12 & $3 b$ & 91 \\
\hline 15 & $3 \mathbf{a}$ & $\mathrm{Bu}_{4} \mathrm{NOH}$ & $\mathrm{Et}_{2} \mathrm{O}$ & 20 & 1 & $3 b$ & 93 \\
\hline
\end{tabular}

Table 1. Desilylation of $\alpha$ trimethylsilylcyclopentenone derivatives $\mathbf{3 a}$ and $\mathbf{8 a}, \mathbf{b}$.

Table 2. 1,4-Addition of organocuprates to $\alpha$-(trimethylsilyl)cyclopentenones 3a and 8a and subsequent desilylation of (trimethylsilyl)ketones $\mathbf{2 , 1 0}$ to the corresponding ketones $\mathbf{1}$ and $\mathbf{1 1}$.

\begin{tabular}{|c|c|c|c|c|c|c|c|c|c|c|}
\hline \multirow{2}{*}{ Entry } & & \multicolumn{2}{|c|}{ Conditions } & \multicolumn{3}{|c|}{ TMS-ketones } & \multicolumn{2}{|c|}{ Conditions } & \multirow{2}{*}{\multicolumn{2}{|c|}{$\begin{array}{l}\text { Ketones } \\
\text { Yield [\%] }\end{array}$}} \\
\hline & & $\mathrm{T}\left[{ }^{\circ} \mathrm{C}\right]$ & $t[\mathrm{~min}]$ & & $\mathrm{R}=$ & Yield [\%] & Solvent & $t[\mathrm{~h}]$ & & \\
\hline$\overline{1}$ & $\mathbf{8 a}$ & 0 & 10 & $\mathbf{1 0 a}$ & $\mathrm{Me}$ & 84 & $\mathrm{THF}$ & 48 & 11a & 98 \\
\hline 2 & $\mathbf{8 a}$ & -20 & 15 & $10 b$ & $\mathrm{Bu}$ & 92 & THF & 12 & 11b & 87 \\
\hline 3 & $8 \mathbf{a}$ & 0 & 120 & $10 \mathrm{c}$ & $\mathrm{Ph}$ & 86 & THF & 1 & 11c & 79 \\
\hline 4 & $\mathbf{8 a}$ & -20 & 30 & 10d & $i$-Pr & 42 & THF & 12 & 11d & 75 \\
\hline 5 & $3 \mathbf{a}$ & 0 & 120 & $2 a$ & $\mathrm{Me}$ & 49 & $\mathrm{Et}_{2} \mathrm{O}$ & 3 & 1a & 63 \\
\hline 6 & $3 \mathbf{a}$ & -20 & 180 & $2 b$ & $\mathrm{Bu}$ & 62 & $\mathrm{Et}_{2} \mathrm{O}$ & 12 & $1 b$ & 77 \\
\hline 7 & $3 \mathbf{a}$ & 0 & 180 & 2c & $\mathrm{Ph}$ & 70 & $\mathrm{Et}_{2} \mathrm{O}$ & 3 & 1c & 90 \\
\hline
\end{tabular}

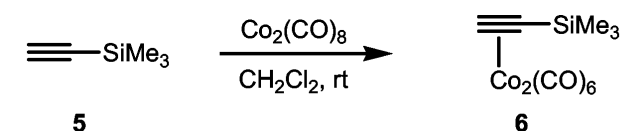

5
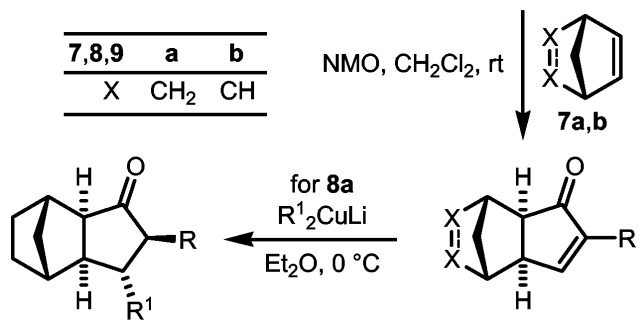

$10 a-d R=$ TMS

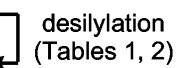

\begin{tabular}{rcccc}
\hline $\mathbf{1 0 , 1 1}$ & a & b & c & d \\
\hline $\mathrm{R}^{1}$ & $\mathrm{Me}$ & $\mathrm{Bu}$ & $\mathrm{Ph}$ & $i-\mathrm{Pr}$ \\
\hline
\end{tabular}

Scheme 2. Reaction sequence of Pauson-Khand reaction, cuprate addition and desilylation using norbornene (7a) and norbornadiene $\mathbf{( 7 b )}$.

Unfortunately, we did not succeed in clean removal of the TMS group by reacting enone $\mathbf{8 a}$ in the presence of TBAF in THF following a known protocol $[6 a, b]$. Therefore, various desilylation condi- tions and reagents such as TASF [9], $\mathrm{CsOH}, \mathrm{LiOH}$, and $\mathrm{MeSO}_{3} \mathrm{H}$ [10] had to be investigated (Table 1). After considerable experimentation, tetrabutylammonium hydroxide $\left(\mathrm{Bu}_{4} \mathrm{NOH}\right)$ [11] in THF was found to be most suitable and the desilylation product 9a was obtained in $98 \%$ yield (entry 9). Analogously norbornadiene-derived $\mathbf{8 b}$ was desilylated to $\mathbf{9 b}$ [11] in $83 \%$ yield (entry 12).

Although the functionalization of norbornadiene-derived $\mathbf{8 b}$ by 1,4-addition of magnesiocuprate reagents was previously reported [6b], we applied the 1,4-addition of lithium dialkylcuprates to norbornene derivative 8a. Subsequently the resulting ketones $\mathbf{1 0}$ were desilylated under the conditions described above (Scheme 2, Table 2). As shown in Table 2, cyclopentenone 8a reacted cleanly with several lithium organocuprates to give the corresponding $\alpha$-trimethylsilyl- $\beta$-substituted ketones $\mathbf{1 0 a}-\mathbf{d}$ in moderate to good yields as single trans diastereoisomers. Subsequent desilylation was achieved uneventfully by treatment with $\mathrm{Bu}_{4} \mathrm{NOH}$ in THF to give products $11 \mathbf{a}-\mathbf{d}$ in $75-98 \%$ yield, thus demonstrating the possibility to remove the trimethylsilyl group even on a late stage of the synthesis. 


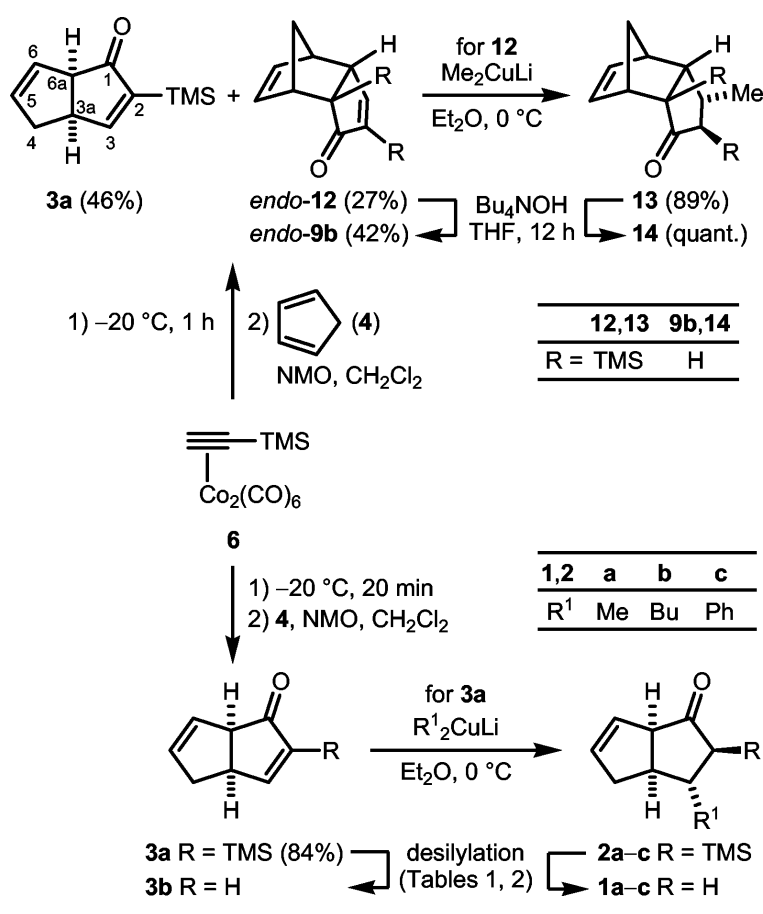

Scheme 3. Synthesis of pentalene derivatives 1 via PausonKhand reaction of cyclopentadiene (4) with trimethylsilylacetylene (5), Michael reaction and desilylation.

The reaction conditions were then applied to the preparation of the desired pentalene derivatives $\mathbf{1}$ (Scheme 3). The Pauson-Khand reaction was slightly modified by cooling the cobalt-alkyne complex 6 to $-20{ }^{\circ} \mathrm{C}$ for $1 \mathrm{~h}$ prior to reaction with cyclopentadiene (4). A mixture of the pentalenone 3a (46\% yield) and a tricyclic endo-cyclopentenone derivative 12 (27\% yield) was isolated (Scheme 3 ). The latter resulted from cocyclization of two equivalents of $\mathbf{5}$ with $\mathrm{Co}_{2}(\mathrm{CO})_{8}$ and subsequent Diels-Alder reaction of the intermediate 2,5-bis(trimethylsilyl)cyclopentadienone with 4 . The endo-configuration of $\mathbf{1 2}$ was confirmed by $\mathrm{X}$-ray crystal structure analysis (Fig. 1). If the cobaltalkyne complex 6 was stirred for only $20 \mathrm{~min}$ at $-20{ }^{\circ} \mathrm{C}$ prior to cyclopentadiene addition, the formation of a single isomer 3a was observed by GC-MS and NMR, and the yield was improved to $84 \%$.

Table 1 reveals that $\mathrm{Et}_{2} \mathrm{O}$ turned out to be the optimal solvent for the desilylation of $\mathbf{3 a}$ giving the product $\mathbf{3 b}$ in $93 \%$ yield (entry 15 ). Thus, enone $\mathbf{3 b}$ was accessible in $78 \%$ total yield via this two-step reaction. As shown in Scheme 3, even endo-cyclopentenone 12 was desilylated to derivative endo-9b [12] by using this system, albeit with lower yield of $42 \%$.

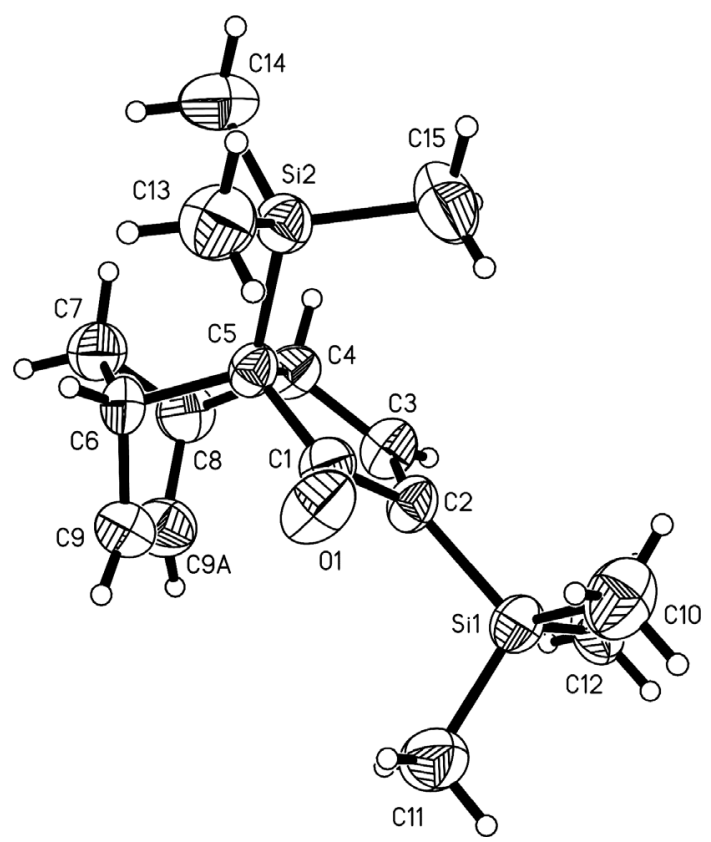

Fig. 1. ORTEP view of the endo-configured tricyclic cyclopentane derivative $\mathbf{1 2}$.

According to our retrosynthetic concept to the target compounds 1 and 2, the $\alpha$-trimethylsilyl-substituted enone 3a was assumed to be a suitable candidate for conjugate 1,4-cuprate addition and was treated with lithium organocuprates under the reaction conditions described above (Scheme 3, Table 2). The addition proceeded cleanly to give the corresponding pentalene derivatives $\mathbf{2 a}-\mathbf{c}$. However, the yields of the conjugate addition to pentalenone $\mathbf{3 a}$ were generally somewhat lower than those of tricyclic enone 8a. Again, the $\alpha$ trimethylsilyl group could be removed at this stage of synthesis with $\mathrm{Bu}_{4} \mathrm{NOH}$ in $\mathrm{Et}_{2} \mathrm{O}$ to afford the functionalized target pentalene systems $1 \mathbf{a}-\mathbf{c}$. The endocompound 12 was reacted in an analogous manner to give both the bis(trimethylsilyl)-substituted methylation product $\mathbf{1 3}$ and the corresponding desilylation product 14 [13] in excellent yields (Scheme 3).

In conclusion, we demonstrated that cyclopentadiene (4) was converted with trimethylsilylacetylene (5) in an intermolecular Pauson-Khand reaction, leading directly to the bicyclic enone $\mathbf{3 a}$ in good yield. As a by-product in the Pauson-Khand reaction an endocoupled enone 12 was isolated. Functionalization of various enones was realized by 1,4-addition of organocuprates, and the desilylation of the enones $\mathbf{3 a}, \mathbf{8}$ or the functionalized ketones $\mathbf{2}, \mathbf{1 0}$ could be performed under mild reaction conditions. A similar reaction se- 
quence was even published for norbornadiene to give tricyclic precursors for cyclopentenone containing natural products [6b, 14], but its application in the synthesis route to functionalized bicyclo[3.3.0] octane derivatives 1 has not been reported so far. Thus, it allows the preparation of compounds $\mathbf{1}$ in only three steps in 26-53\% total yield, starting with an intermolecular Pauson-Khand reaction of cyclopentadiene as the key step. The pentalenones $\mathbf{1 - 3}$ may be further functionalized at the alkene moiety.

\section{Experimental Section}

\section{General information}

Column chromatography was accomplished using $\mathrm{SiO}_{2}$ 60 , grain size $0.063-0.200 \mathrm{~mm}$ (Merck) with pentane, hexanes (b. p. $\left.40-60{ }^{\circ} \mathrm{C}\right)$, EtOAc, and diethyl ether $\left(\mathrm{Et}_{2} \mathrm{O}\right)$ as eluents. All starting materials are commercially available. The following spectroscopic and analytical instruments were used. IR: Bruker Vektor22 FTIR. - NMR: Bruker ARX 300 and Avance $500\left({ }^{1} \mathrm{H}: 300.13 \mathrm{MHz}, 500.15 \mathrm{MHz},{ }^{13} \mathrm{C}\right.$ : 75.47 MHz, $125.76 \mathrm{MHz}$ ). For ${ }^{1} \mathrm{H}$ spectra, TMS was used as internal standard. For ${ }^{13} \mathrm{C}$ spectra, signal assignments are based on DEPT experiments. - Melting points: Mettler Toledo DSC 822e, m. p. are uncorrected. - Mass spectrometry: Finnigan MAT 95 and Varian MAT 711.

Pauson-Khand reaction of cyclopentadiene (4) with $\mathbf{5}$ to derivatives $\mathbf{3 a}$ and $\mathbf{1 2}$

TMS-acetylene 5 (705 $\mu 1,5.0 \mathrm{mmol})$ was added to a solution of dicobaltoctacarbonyl $(1.86 \mathrm{~g}, 5.4 \mathrm{mmol})$ in $\mathrm{CH}_{2} \mathrm{Cl}_{2}$ $(25 \mathrm{ml})$ under $\mathrm{N}_{2}$ atmosphere and the reaction mixture stirred at $-20{ }^{\circ} \mathrm{C}$ for $1 \mathrm{~h}$. Then $4(700 \mu \mathrm{l}, 7.7 \mathrm{mmol})$ was added dropwise, and the reaction mixture stirred for a further $20 \mathrm{~min}$ at $-20^{\circ} \mathrm{C}$. $N$-methylmorpholin- $N$-oxide (NMO) $(3.525 \mathrm{~g}, 5.0 \mathrm{mmol})$ was added portionwise and after stirring for $6 \mathrm{~h}$, the reaction mixture was filtered through $\mathrm{SiO}_{2}$ with $\mathrm{Et}_{2} \mathrm{O}$. The filtrate was concentrated and the residue chromatographed on $\mathrm{SiO}_{2}$ with pentane/Et $2 \mathrm{O}(40: 1)$ to give in a first fraction $\left(R_{f}=0.27\right)$ compound $12(426 \mathrm{mg}, 1.34 \mathrm{mmol}$, $27 \%)$ as a colorless solid and in a second fraction $\left(R_{f}=0.24\right)$ compound 3a (444 mg, $2.31 \mathrm{mmol}, 46 \%)$ as a colorless crystalline solid.

rac-2,7a-Bis(trimethylsilyl)-3a,4,7,7a-tetrahydro-1H-4,7methanoinden-1-one (12)

M. p. $75-78{ }^{\circ} \mathrm{C} .-$ FT-IR (ATR): $v=3069(\mathrm{w}), 3025(\mathrm{~m})$, 2979 (s), 2952 (m), 1655 (vs), 1572 (s), 1294 (s), 1247 (s), $1188(\mathrm{~s}), 988(\mathrm{~m}) \mathrm{cm}^{-1} .-{ }^{1} \mathrm{H}$ NMR $\left(500.15 \mathrm{MHz}, \mathrm{CDCl}_{3}\right)$ : $\delta=0.07$ (s, $\left.9 \mathrm{H}, \mathrm{CHSi}\left(\mathrm{CH}_{3}\right)_{3}\right), 0.08\left(\mathrm{~s}, 9 \mathrm{H}, \mathrm{CSi}\left(\mathrm{CH}_{3}\right)_{3}\right)$, $1.51\left(\mathrm{dt}, J=1.6 \mathrm{~Hz}, J=8.3 \mathrm{~Hz}, 1 \mathrm{H}, 10-\mathrm{H}_{\mathrm{b}}\right), 1.67(\mathrm{~d}$,
$\left.J=8.3 \mathrm{~Hz}, 1 \mathrm{H}, 10-\mathrm{H}_{\mathrm{a}}\right), 2.93-2.97(\mathrm{~m}, 1 \mathrm{H}, 1-\mathrm{H}), 3.07-$ $3.09(\mathrm{~m}, 1 \mathrm{H}, 7-\mathrm{H}), 3.37(\mathrm{dd}, J=3.0 \mathrm{~Hz}, J=3.9 \mathrm{~Hz}$, $1 \mathrm{H}, 6-\mathrm{H}), 5.68(\mathrm{dd}, J=3.0 \mathrm{~Hz}, J=5.4 \mathrm{~Hz}, 1 \mathrm{H}, 9-$ H), $5.89(\mathrm{dd}, J=2.8 \mathrm{~Hz}, J=5.4 \mathrm{~Hz}, 1 \mathrm{H}, 8-\mathrm{H}), 7.54$ $(\mathrm{d}, J=2.7 \mathrm{~Hz}, 1 \mathrm{H}, 5-\mathrm{H}) .-{ }^{13} \mathrm{C}$ NMR $(125.76 \mathrm{MHz}$, $\left.\mathrm{CDCl}_{3}\right): \delta=-0.6\left(\left(\mathrm{CH}_{3}\right)_{3} \mathrm{Si}\right),-0.9\left(\left(\mathrm{CH}_{3}\right)_{3} \mathrm{Si}\right), 46.4,48.6$ (C-8, C-9), 52.6 (C-10), 55.1 (C-6), 55.6 (C-2), 132.4, 137.4 (C-8, C-9), 150.9 (C-4), 171.5 (C-5), 217.1 (C-3). - GCMS (EI): $m / z$ (\%) 290.2 (17) $\left[\mathrm{M}^{+}\right], 259.2$ (10), 209.1 (23), 202.2 (17), 193.1 (15), 147.1 (13), 133.0 (13), 73.0 (100), 66.0 (31). - HRMS (EI): calcd. for $\mathrm{C}_{16} \mathrm{H}_{26} \mathrm{OSi}_{2} 290.1522$; found $290.1516\left[\mathrm{M}^{+}\right]$.

\section{rac-2-(Trimethylsilyl)-4,6a-dihydropentalen-1(3aH)-one} (3a)

M.p. $37{ }^{\circ}$ C. - FT-IR (ATR): $v=3061$ (w), 3033 (w), $2960(\mathrm{~m}), 2933(\mathrm{~m}), 2901(\mathrm{~m}), 2848(\mathrm{~m}), 2360(\mathrm{w})$, 1685 (vs), 1573 (s), 1293 (s), 1277 (s), 1245 (s), 1214 (s), 1176 (s), 971 (m), 831 (s), 814 (s), 749 (s), 678 (s) $\mathrm{cm}^{-1} .-{ }^{1} \mathrm{H}$ NMR $\left(500.15 \mathrm{MHz}, \mathrm{CDCl}_{3}\right): \delta=0.13(\mathrm{~s}, 9 \mathrm{H}$, $\left.\mathrm{Si}\left(\mathrm{CH}_{3}\right)_{3}\right), 2.27$ (tdd, $J=2.6 \mathrm{~Hz}, J=5.4 \mathrm{~Hz}, J=17.6 \mathrm{~Hz}$, $\left.1 \mathrm{H}, 4-\mathrm{H}_{\mathrm{b}}\right), 2.65-2.73\left(\mathrm{~m}, 1 \mathrm{H}, 4-\mathrm{H}_{\mathrm{a}}\right), 3.32-3.37(\mathrm{~m}, 1 \mathrm{H}$, $3 \mathrm{a}-\mathrm{H}), 3.57$ (tdd, $J=2.8 \mathrm{~Hz}, J=5.7 \mathrm{~Hz}, J=11.1 \mathrm{~Hz}$, $1 \mathrm{H}, 6 \mathrm{a}-\mathrm{H}), 5.56(\mathrm{td}, J=2.4 \mathrm{~Hz}, J=7.8 \mathrm{~Hz}, 1 \mathrm{H}, 5-$ $\mathrm{H}), 5.63(\mathrm{td}, J=2.4 \mathrm{~Hz}, J=7.9 \mathrm{~Hz}, 1 \mathrm{H}, 6-\mathrm{H}), 7.72(\mathrm{~d}$, $J=2.5 \mathrm{~Hz}, 1 \mathrm{H}, 3-\mathrm{H}) .-{ }^{13} \mathrm{C} \mathrm{NMR}\left(125.76 \mathrm{MHz}, \mathrm{CDCl}_{3}\right)$ : $\delta=-0.8\left(\left(\mathrm{CH}_{3}\right)_{3} \mathrm{Si}\right), 37.3(\mathrm{C}-4), 46.6(\mathrm{C}-3 \mathrm{a}), 60.9(\mathrm{C}-6 \mathrm{a})$, 130.6, 132.2 (C-5, C-6), 144.7 (C-2), 176.2 (C-3), 213.3 (C-1). - GC-MS (EI): $m / z$ (\%) $192.1(64)\left[\mathrm{M}^{+}\right], 177.1$ (73), 149.1 (17), 99.0 (15), 73.0 (100), 66.0 (18). - HRMS (EI): calcd. for $\mathrm{C}_{11} \mathrm{H}_{16} \mathrm{OSi}$ 192.0970; found $192.0967\left[\mathrm{M}^{+}\right]$.

\section{Preparation of $\mathbf{3 a}$}

TMS-acetylene (5) $(141 \mu \mathrm{l}, 1.0 \mathrm{mmol})$ was added to a solution of dicobaltoctacarbonyl $(342 \mathrm{mg}, 1.0 \mathrm{mmol})$ in $\mathrm{CH}_{2} \mathrm{Cl}_{2}(5 \mathrm{ml})$ under $\mathrm{N}_{2}$ atmosphere and the reaction mixture stirred at $-20{ }^{\circ} \mathrm{C}$ for $20 \mathrm{~min}$. Then $4(90 \mu 1,1.0 \mathrm{mmol})$ was added dropwise, and the reaction mixture stirred for a further $20 \mathrm{~min}$ at $-20{ }^{\circ} \mathrm{C}$. NMO (705 g, $\left.1.0 \mathrm{mmol}\right)$ was added portionwise and after stirring for $6 \mathrm{~h}$, the reaction mixture was filtered through $\mathrm{SiO}_{2}$ with $\mathrm{Et}_{2} \mathrm{O}$. The filtrate was concentrated and the residue chromatographed on $\mathrm{SiO}_{2}$ with pentane $/ \mathrm{Et}_{2} \mathrm{O}(40: 1)$ to give $\mathbf{3 a}(162 \mathrm{mg}, 0.84 \mathrm{mmol}, 84 \%)$.

\section{Pauson-Khand reaction of alkenes $\mathbf{7 a}, \mathbf{b}$ with $\mathbf{5}$}

TMS-acetylene (5) was added to a solution of dicobaltoctacarbonyl (342 mg, $1.0 \mathrm{mmol}$ ) in $\mathrm{CH}_{2} \mathrm{Cl}_{2}(5 \mathrm{ml})$ under $\mathrm{N}_{2}$ atmosphere and the reaction mixture stirred at $\mathrm{r}$. t. for $20 \mathrm{~min}$. Then alkene $7 \mathbf{a}$ or $7 \mathbf{b}(1.2 \mathrm{mmol})$ was added dropwise, and the reaction mixture stirred at $r$. t. for a further $20 \mathrm{~min}$. NMO (705 mg, $6.0 \mathrm{mmol}$ ) was added portionwise and after stir- 
ring for $30 \mathrm{~min}$, the reaction mixture was filtered through $\mathrm{SiO}_{2}$ with $\mathrm{Et}_{2} \mathrm{O}$. The filtrate was concentrated and the residue chromatographed on $\mathrm{SiO}_{2}$ with pentane/Et ${ }_{2} \mathrm{O}$ to give products $8 \mathbf{a}$ or $\mathbf{8 b}$. Physical and spectroscopic data were in accordance with those in the literature [8].

\section{General procedure for the cuprate addition}

A suspension of copper(II) iodide $(104 \mathrm{mg}, 0.54 \mathrm{mmol})$ in $\mathrm{Et}_{2} \mathrm{O}(5 \mathrm{ml})$ was stirred at $0{ }^{\circ} \mathrm{C}$ or $-20{ }^{\circ} \mathrm{C}$ (see Table 2) for $10 \mathrm{~min}$. Then the respective organolithium compound $(1.08 \mathrm{mmol})$ was added dropwise and the reaction mixture stirred for a further $10 \mathrm{~min}$ at the given temperature. The respective enone 3a, 12, or $\mathbf{8 a}(0.45 \mathrm{mmol})$ was added, the reaction mixture stirred for $10-180 \mathrm{~min}$ (tlc control) and then hydrolyzed with a saturated solution of $\mathrm{NH}_{4} \mathrm{Cl}(5 \mathrm{ml})$. The layers were separated and the aqueous layer was extracted with $\mathrm{Et}_{2} \mathrm{O}(5 \times 10 \mathrm{ml})$. The combined organic layers were dried $\left(\mathrm{K}_{2} \mathrm{CO}_{3}\right)$, concentrated and the crude products 2,10 , and 13 purified by chromatography on $\mathrm{SiO}_{2}$ with pentane/Et $2 \mathrm{O}(20: 1)$ or $(10: 1)$ for 10a, d, 13.

rac-3-Methyl-2-(trimethylsilyl)-3,3a,4,6a-tetrahydropentalen-1(2H)-one (2a)

$R_{f}=0.24 .-$ FT-IR (ATR): $v=2952(\mathrm{~s}), 2924(\mathrm{~s})$, $2900(\mathrm{~m}), 2870(\mathrm{~m}), 2848(\mathrm{~m}), 1706$ (vs), $1457(\mathrm{~m})$, 1444 (m), 1416 (w), 1377 (m), 1247 (vs), 1173 (s), 916 (s), $836(\mathrm{~s}) \mathrm{cm}^{-1} \cdot-{ }^{1} \mathrm{H}$ NMR $\left(500.15 \mathrm{MHz},\left(\mathrm{CD}_{3}\right)_{2} \mathrm{CO}\right): \delta=$ $0.03\left(\mathrm{~s}, 9 \mathrm{H}, \mathrm{Si}\left(\mathrm{CH}_{3}\right)_{3}\right), 1.15\left(\mathrm{~d}, J=6.8 \mathrm{~Hz}, 3 \mathrm{H}, \mathrm{CH}_{3}\right)$, $1.64(\mathrm{dd}, J=1.7 \mathrm{~Hz}, J=10.0 \mathrm{~Hz}, 1 \mathrm{H}, 2-\mathrm{H}), 1.76-$ $1.85(\mathrm{~m}, 1 \mathrm{H}, 3-\mathrm{H}), 2.26-2.32\left(\mathrm{~m}, 1 \mathrm{H}, 4-\mathrm{H}_{\mathrm{a}}\right), 2.38$ (q, $J=7.6 \mathrm{~Hz}, 1 \mathrm{H}, 3 \mathrm{a}-\mathrm{H}), 2.57-2.64\left(\mathrm{~m}, 1 \mathrm{H}, 4-\mathrm{H}_{\mathrm{b}}\right), 3.27-$ $3.32(\mathrm{~m}, 1 \mathrm{H}, 6 \mathrm{a}-\mathrm{H}), 5.46(\mathrm{td}, J=2.3 \mathrm{~Hz}, J=7.2 \mathrm{~Hz}$, $1 \mathrm{H}, 5-\mathrm{H}), 5.81(\mathrm{dt}, J=2.6 \mathrm{~Hz}, J=5.2 \mathrm{~Hz}, 1 \mathrm{H}, 6-\mathrm{H})$. ${ }^{13} \mathrm{C}$ NMR $\left(125.76 \mathrm{MHz},\left(\mathrm{CD}_{3}\right)_{2} \mathrm{CO}\right): \delta=-0.8\left(\mathrm{Si}\left(\mathrm{CH}_{3}\right)_{3}\right)$, $23.1\left(\mathrm{CH}_{3}\right), 41.1$ (C-4), 42.3 (C-3), 49.7 (C-3a), 50.7 (C-2), 65.3 (C-6a), 129.1 (C-5), 134.1 (C-6). - MS (EI): $\mathrm{m} / z$ (\%) $208.1(32)\left[\mathrm{M}^{+}\right], 193.1$ (100), 180.1 (6), 79.1 (14), 73.1 (75), 66.1 (9), 45.0 (8). - HRMS (EI): calcd. for $\mathrm{C}_{12} \mathrm{H}_{20} \mathrm{OSi}$ 208.1283; found $208.1291\left[\mathrm{M}^{+}\right]$.

rac-3-Butyl-2-(trimethylsilyl)-3,3a,4,6a-tetrahydropentalen1(2H)-one (2b)

$R_{f}=0.20 .-$ FT-IR (ATR): $v=2955(\mathrm{~s}), 2925(\mathrm{~s})$, 2857 (m), 1706 (vs), 1466 (m), 1378 (w), 1248 (vs), 1152 (s), $1058(\mathrm{~m}), 1022(\mathrm{~m}), 918(\mathrm{~s}), 891(\mathrm{~m}), 725(\mathrm{~m}), 693(\mathrm{~s})$ $\mathrm{cm}^{-1} .-{ }^{1} \mathrm{H}$ NMR $\left(500.15 \mathrm{MHz}, \mathrm{CDCl}_{3}\right): \delta=0.04(\mathrm{~s}, 9 \mathrm{H}$, $\left.\mathrm{Si}\left(\mathrm{CH}_{3}\right)_{3}\right), 0.91$ (t, $\left.J=7.0 \mathrm{~Hz}, 3 \mathrm{H}, \mathrm{CH}_{3}\right), 1.23-1.45$ (m, $\left.5 \mathrm{H}, 1^{\prime}-\mathrm{H}_{\mathrm{a}}, 2^{\prime}-\mathrm{H}, 3^{\prime}-\mathrm{H}\right), 1.47-1.55\left(\mathrm{~m}, 1 \mathrm{H}, 1^{\prime}-\mathrm{H}_{\mathrm{b}}\right), 1.72$ $(\mathrm{dd}, J=1.9 \mathrm{~Hz}, J=7.7 \mathrm{~Hz}, 1 \mathrm{H}, 2-\mathrm{H}), 1.80-1.86(\mathrm{~m}, 1 \mathrm{H}$, $3-\mathrm{H}), 2.26\left(\mathrm{~d}, J=17.0 \mathrm{~Hz}, 1 \mathrm{H}, 4-\mathrm{H}_{\mathrm{a}}\right), 2.51-2.56(\mathrm{~m}, 1 \mathrm{H}$, $3 \mathrm{a}-\mathrm{H}$ ), 2.72 (dddd, $J=2.6 \mathrm{~Hz}, J=4.9 \mathrm{~Hz}, J=7.8 \mathrm{~Hz}$,
$\left.J=16.7 \mathrm{~Hz}, 1 \mathrm{H}, 4-\mathrm{H}_{\mathrm{b}}\right), 3.37-3.42(\mathrm{~m}, 1 \mathrm{H}, 6 \mathrm{a}-\mathrm{H}), 5.50(\mathrm{td}$, $J=2.3 \mathrm{~Hz}, J=7.4 \mathrm{~Hz}, 1 \mathrm{H}, 5-\mathrm{H}), 5.78(\mathrm{dt}, J=2.5 \mathrm{~Hz}, J=$ $5.2 \mathrm{~Hz}, 1 \mathrm{H}, 6-\mathrm{H}) .-{ }^{13} \mathrm{C} \mathrm{NMR}\left(125.76 \mathrm{MHz}, \mathrm{CDCl}_{3}\right): \delta=$ $-1.8\left(\mathrm{Si}\left(\mathrm{CH}_{3}\right)_{3}\right), 14.0$ (C-4'), 22.9 (C-3'), 29.4 (C-2'), 38.4 (C-1'), 41.8 (C-4), 45.2 (C-3a), 45.9 (C-3), 48.8 (C-2), 63.6 (C-6a), 127.5 (C-5), 132.5 (C-6). - GC-MS (EI): $\mathrm{m} / \mathrm{z}(\%)$ $250.4(4)\left[\mathrm{M}^{+}\right], 193.2(100), 183.2$ (15), 73.1 (61), 55.1 (6). HRMS (CI, $\mathrm{CH}_{4}$ ): calcd. for $\mathrm{C}_{15} \mathrm{H}_{25} \mathrm{OSi} 249.1674$; found $249.1666[\mathrm{M}-\mathrm{H}]^{+}$.

rac-3-Phenyl-2-(trimethylsilyl)-3,3a,4,6a-tetrahydropentalen-1(2H)-one (2c)

$R_{f}=0.27 .-$ M.p. $102^{\circ} \mathrm{C} .-$ FT-IR (ATR): $v=3059(\mathrm{~m})$, 3026 (w), 2950 (s), 2922 (s), 2891 (s), 2852 (m), 1699 (vs), 1599 (s), 1491 (s), 1452 (s), 1443 (s), 1360 (w), 1263 (s), 1247 (vs), 1224 (s), 1175 (s), 1159 (s), 1143 (s), 1074 (s), 1037 (m), 1029 (w), 946 (s), 924 (s), 910 (s) $\mathrm{cm}^{-1}$. ${ }^{1} \mathrm{H}$ NMR $\left(500.15 \mathrm{MHz},\left(\mathrm{CD}_{3}\right)_{2} \mathrm{CO}\right): \delta=-0.11$ (s, $9 \mathrm{H}$, $\left.\mathrm{Si}\left(\mathrm{CH}_{3}\right)_{3}\right), 2.35(\mathrm{dd}, J=1.8 \mathrm{~Hz}, J=11.3 \mathrm{~Hz}, 1 \mathrm{H}, 2-\mathrm{H})$, $2.37-2.43\left(\mathrm{~m}, 1 \mathrm{H}, 4-\mathrm{H}_{\mathrm{a}}\right), 2.43-2.49\left(\mathrm{~m}, 1 \mathrm{H}, 4-\mathrm{H}_{\mathrm{b}}\right), 2.79-$ $2.84(\mathrm{~m}, 1 \mathrm{H}, 3 \mathrm{a}-\mathrm{H}), 2.89$ (dd, $J=8.6 \mathrm{~Hz}, J=11.2 \mathrm{~Hz}$, $1 \mathrm{H}, 3-\mathrm{H}), 3.46-3.51(\mathrm{~m}, 1 \mathrm{H}, 6 \mathrm{a}-\mathrm{H}), 5.55(\mathrm{td}, J=2.2 \mathrm{~Hz}$, $J=7.2 \mathrm{~Hz}, 1 \mathrm{H}, 5-\mathrm{H}), 5.87(\mathrm{dt}, J=2.6 \mathrm{~Hz}, J=5.3 \mathrm{~Hz}, 1 \mathrm{H}$, 6-H), 7.21-7.25 (m, 1 H, 4'-H), $7.31-7.43$ (m, 4 H, 2'-H, 3'-H, 5'-H, 6'-H). - ${ }^{13} \mathrm{C}$ NMR (125.76 MHz, $\left.\left(\mathrm{CD}_{3}\right)_{2} \mathrm{CO}\right)$ : $\delta=-0.9\left(\mathrm{Si}\left(\mathrm{CH}_{3}\right)_{3}\right), 40.0(\mathrm{C}-4), 50.7(\mathrm{C}-2), 51.5(\mathrm{C}-3 \mathrm{a})$, 53.8 (C-3), 65.0 (C-6a), 128.2 (C-4'), 128.9 (C-5), 129.7, 130.3 (C-2', C-3', C-5', C-6'), 133.9 (C-6), 147.4 (C-1'), 219.1 (C-1). - GC-MS (EI): $m / z$ (\%) 270.2 (72) [M ], 255.2 (10), 242.2 (19), 229.2 (8), 193.2 (9), 179.2 (32), 165.2 (21), 153.2 (10), 115.1 (12), 103.0 (22), 73.0 (100), 66.1 (12), 50.9 (7). - HRMS (EI): calcd. for $\mathrm{C}_{17} \mathrm{H}_{22} \mathrm{OSi} 270.1440$; found $270.1441\left[\mathrm{M}^{+}\right]$.

rac-3-Methyl-2-(trimethylsilyl)octahydro-1H-4,7-methanoinden-1-one (10a)

$R_{f}=0.35 .-$ FT-IR (ATR): $v=2949(\mathrm{~s}), 2911(\mathrm{~m})$, 2871 (m), 1708 (vs), 1475 (m), 1375 (w), 1288 (w), 1246 (s), 1207 (s), 1168 (s), 1134 (s), 1101 (s), 1017 (m), 981 (m) $\mathrm{cm}^{-1}$. $-{ }^{1} \mathrm{H}$ NMR $\left(300.13 \mathrm{MHz}, \mathrm{CDCl}_{3}\right): \delta=0.08(\mathrm{~s}$, $\left.9 \mathrm{H}, \mathrm{Si}\left(\mathrm{CH}_{3}\right)_{3}\right), 0.93-1.05(\mathrm{~m}, 2 \mathrm{H}, 10-\mathrm{H}), 1.11(\mathrm{~d}, J=$ $\left.1.9 \mathrm{~Hz}, 1 \mathrm{H}, 8-\mathrm{H}_{\mathrm{a}}\right), 1.14\left(\mathrm{~d}, J=1.9 \mathrm{~Hz}, 1 \mathrm{H}, 9-\mathrm{H}_{\mathrm{a}}\right), 1.17$ (d, $\left.J=6.4 \mathrm{~Hz}, 3 \mathrm{H}, \mathrm{CH}_{3}\right), 1.41-1.57(\mathrm{~m}, 3 \mathrm{H}, 6-\mathrm{H}, 8-$ $\left.\mathrm{H}_{\mathrm{b}}, 9-\mathrm{H}_{\mathrm{b}}\right), 1.67-1.77(\mathrm{~m}, 2 \mathrm{H}, 2-\mathrm{H}, 5-\mathrm{H}), 2.14-2.22(\mathrm{~m}$, $2 \mathrm{H}, 1-\mathrm{H}, 4-\mathrm{H}), 2.47-2.51(\mathrm{~m}, 1 \mathrm{H}, 7-\mathrm{H}) .-{ }^{13} \mathrm{C} \mathrm{NMR}$ (75.47 MHz, $\left.\mathrm{CDCl}_{3}\right): \delta=-1.6\left(\mathrm{Si}\left(\mathrm{CH}_{3}\right)_{3}\right), 23.9\left(\mathrm{CH}_{3}\right)$, 28.0, 28.6 (C-8, C-9), 34.7 (C-10), 38.2 (C-5), 40.4 (C-7), 42.6 (C-1), 49.4 (C-6), 53.5 (C-2), 58.8 (C-4). - GC-MS (EI): $\mathrm{m} / \mathrm{z}(\%) 236(10)\left[\mathrm{M}^{+}\right], 221$ (100), 169 (5), 155 (4), 91 (7), 73 (78), 67 (12), 45 (17). HRMS (EI): calcd. for $\mathrm{C}_{14} \mathrm{H}_{24} \mathrm{OSi}$ 236.1596, found $236.1596\left[\mathrm{M}^{+}\right]$. 
rac-3-Butyl-2-(trimethylsilyl)octahydro-1H-4,7-methanoinden-1-one (10b)

$R_{f}=0.30 .-$ FT-IR (ATR): $v=2951(\mathrm{~s}), 2926(\mathrm{~s})$, 2871 (m), 1708 (vs), 1457 (m), 1378 (w), 1246 (s), 1200 (s), 1184 (m), 1167 (s), 1130 (s), 1102 (s), 1050 (w), 1005 (w), $936(\mathrm{~m}) \mathrm{cm}^{-1} .-{ }^{1} \mathrm{H}$ NMR $\left(500.15 \mathrm{MHz},\left(\mathrm{CD}_{3}\right)_{2} \mathrm{CO}\right): \delta=$ 0.08 (s, $\left.9 \mathrm{H}, \mathrm{Si}\left(\mathrm{CH}_{3}\right)_{3}\right), 0.93$ (t, $\left.J=6.7 \mathrm{~Hz}, 3 \mathrm{H}, 4^{\prime}-\mathrm{H}\right)$, $0.98-1.05(\mathrm{~m}, 2 \mathrm{H}, 10-\mathrm{H}), 1.11-1.16\left(\mathrm{~m}, 1 \mathrm{H}, 8-\mathrm{H}_{\mathrm{a}}\right), 1.18-$ $1.24\left(\mathrm{~m}, 1 \mathrm{H}, 9-\mathrm{H}_{\mathrm{a}}\right), 1.29-1.43\left(\mathrm{~m}, 4 \mathrm{H}, 1^{\prime}-\mathrm{H}_{\mathrm{a}}, 2^{\prime}-\mathrm{H}_{\mathrm{a}}, 3^{\prime}-\mathrm{H}\right)$, $1.45-1.52\left(\mathrm{~m}, 3 \mathrm{H}, 2^{\prime}-\mathrm{H}_{\mathrm{b}}, 8-\mathrm{H}_{\mathrm{b}}, 9-\mathrm{H}_{\mathrm{b}}\right), 1.60-1.72(\mathrm{~m}, 3 \mathrm{H}$, 1'- $\left.\mathrm{H}_{\mathrm{b}}, 5-\mathrm{H}, 6-\mathrm{H}\right), 1.86$ (dd, $J=5.1 \mathrm{~Hz}, J=8.9 \mathrm{~Hz}, 1 \mathrm{H}$, 2-H), 2.15 (d, $J=9.5 \mathrm{~Hz}, 1 \mathrm{H}, 4-\mathrm{H}), 2.16-2.19(\mathrm{~m}, 1 \mathrm{H}$, 1-H), $2.38-2.42(\mathrm{~m}, 1 \mathrm{H}, 7-\mathrm{H}) .-{ }^{13} \mathrm{C}$ NMR $(125.76 \mathrm{MHz}$, $\left.\left(\mathrm{CD}_{3}\right)_{2} \mathrm{CO}\right): \delta=-0.4\left(\mathrm{Si}\left(\mathrm{CH}_{3}\right)_{3}\right), 15.4\left(\mathrm{C}-4^{\prime}\right), 24.5\left(\mathrm{C}-3^{\prime}\right)$, 29.7, 30.0 (C-8, C-9), 31.4 (C-2'), 36.0 (C-10), 40.2 (C-1'), 42.1 (C-7), 44.9 (C-5), 45.7 (C-1), 49.1 (C-6), 53.1 (C-2), 60.1 (C-4). - GC-MS (EI): $m / z$ (\%) 278 (2) [M+ $\mathrm{M}^{+}, 263$ (4), 221 (100), 73 (44), 67 (7), 45 (9). $-\mathrm{C}_{17} \mathrm{H}_{30} \mathrm{OSi}$ (278.5): calcd. C 73.31, H 10.86; found C 73.31, H 10.82 .

rac-3-Phenyl-2-(trimethylsilyl)octahydro-1H-4,7-methanoinden-1-one $(\mathbf{1 0 c})$

$R_{f}=0.37 .-$ FT-IR (ATR): $v=3063(\mathrm{w}), 2948(\mathrm{~s})$, 2940 (s), 2876 (m), 2847 (m), 1704 (vs), 1598 (m), $1492(\mathrm{~m}), 1451$ (m), 1240 (s), 1197 (s), 1178 (s), 1132 (m), 1121 (m), 1099 (s), 1028 (m), 1015 (m) cm ${ }^{-1} .-{ }^{1} \mathrm{H}$ NMR (500.15 MHz, $\left.\left(\mathrm{CD}_{3}\right)_{2} \mathrm{CO}\right): \delta=-0.09\left(\mathrm{~s}, 9 \mathrm{H}, \mathrm{Si}\left(\mathrm{CH}_{3}\right)_{3}\right)$, $1.00-1.19\left(\mathrm{~m}, 4 \mathrm{H}, 8-\mathrm{H}_{\mathrm{a}}, 9-\mathrm{H}_{\mathrm{a}}, 10-\mathrm{H}\right), 1.48-1.52(\mathrm{~m}$, $\left.2 \mathrm{H}, 8-\mathrm{H}_{\mathrm{b}}, 9-\mathrm{H}_{\mathrm{b}}\right), 2.12(\mathrm{dd}, J=6.8 \mathrm{~Hz}, J=9.2 \mathrm{~Hz}$, $1 \mathrm{H}, 6-\mathrm{H}), 2.29-2.33(\mathrm{~m}, 2 \mathrm{H}, 4-\mathrm{H}, 7-\mathrm{H}), 2.37$ (d, $J=$ $9.5 \mathrm{~Hz}, 1 \mathrm{H}, 2-\mathrm{H}), 2.48-2.51(\mathrm{~m}, 1 \mathrm{H}, 1-\mathrm{H}), 2.83(\mathrm{dd}, J=$ $9.2 \mathrm{~Hz}, J=21.2 \mathrm{~Hz}, 1 \mathrm{H}, 5-\mathrm{H}), 7.18-7.23\left(\mathrm{~m}, 1 \mathrm{H}, 4^{\prime}-\mathrm{H}\right)$, 7.30-7.42 (m, 4 H, 2'-H, 3'-H, 5'-H, 6'-H). ${ }^{13}{ }^{13} \mathrm{C}$ NMR $\left(125.76 \mathrm{MHz},\left(\mathrm{CD}_{3}\right)_{2} \mathrm{CO}\right): \delta=-0.7\left(\mathrm{Si}\left(\mathrm{CH}_{3}\right)_{3}\right), 29.5,29.9$ (C-8, C-9), 36.0 (C-10), 41.9 (C-1), 43.7 (C-7), 49.8 (C-4), 51.7 (C-5), 56.9 (C-6), 59.8 (C-2), 128.0 (C-4'), 129.3, 130.3 (C-2', C-3', C-5', C-6'), 149.1 (C-1'). - MS (EI): m/z (\%) $298.2(80)\left[\mathrm{M}^{+}\right], 297.2$ (100), 257.2 (8), 231.1 (49), 203.1 (6), 161.1 (9), 135.1 (7), 91.0 (8), 73.1 (67), 59.1 (6), 45.0 (5). - HRMS (EI): calcd. for $\mathrm{C}_{19} \mathrm{H}_{26} \mathrm{OSi} 298.1753$; found $298.1737\left[\mathrm{M}^{+}\right]$.

rac-3-Isopropyl-2-(trimethylsilyl)octahydro-1H-4,7-methanoinden-1-one (10d)

$R_{f}=0.50 .-$ FT-IR (ATR): $v=2951$ (s), 2871 (s), 1706 (vs), $1464(\mathrm{~m}), 1409(\mathrm{~m}), 1246(\mathrm{~s}), 1205$ (s), $1170(\mathrm{~s})$, 1138 (s), 1123 (s), $1103(\mathrm{~m}), 1060(\mathrm{~m}), 1038(\mathrm{w}), 1014(\mathrm{w})$, $998(\mathrm{~m}) \mathrm{cm}^{-1} .-{ }^{1} \mathrm{H}$ NMR $\left(500.15 \mathrm{MHz},\left(\mathrm{CD}_{3}\right)_{2} \mathrm{CO}\right): \delta=$ 0.09 (s, $\left.9 \mathrm{H}, \mathrm{Si}\left(\mathrm{CH}_{3}\right)_{3}\right), 0.88\left(\mathrm{~d}, J=6.5 \mathrm{~Hz}, 3 \mathrm{H}, \mathrm{CH}_{3}\right)$, $1.01\left(\mathrm{~d}, J=6.8 \mathrm{~Hz}, 3 \mathrm{H}, \mathrm{CH}_{3}\right), 1.02-1.07(\mathrm{~m}, 2 \mathrm{H}, 10-\mathrm{H})$, $1.12-1.28\left(\mathrm{~m}, 2 \mathrm{H}, 8-\mathrm{H}_{\mathrm{a}}, 9-\mathrm{H}_{\mathrm{a}}\right), 1.44-1.54\left(\mathrm{~m}, 2 \mathrm{H}, 8-\mathrm{H}_{\mathrm{b}}\right.$,
9- $\left.\mathrm{H}_{\mathrm{b}}\right), 1.77-1.84$ (m, $\left.3 \mathrm{H}, 2-\mathrm{H}, 6-\mathrm{H}, \mathrm{CH}\left(\mathrm{CH}_{3}\right)_{2}\right), 1.96$ (d, $J=9.1 \mathrm{~Hz}, 1 \mathrm{H}, 5-\mathrm{H}), 2.04-2.09(\mathrm{~m}, 1 \mathrm{H}, 4-\mathrm{H}), 2.10-$ $2.13(\mathrm{~m}, 1 \mathrm{H}, 1-\mathrm{H}), 2.37-2.40(\mathrm{~m}, 1 \mathrm{H}, 7-\mathrm{H}) .-{ }^{13} \mathrm{C} \mathrm{NMR}$ $\left(125.76 \mathrm{MHz},\left(\mathrm{CD}_{3}\right)_{2} \mathrm{CO}\right): \delta=-0.1\left(\mathrm{Si}\left(\mathrm{CH}_{3}\right)_{3}\right), 17.2$ $\left(\mathrm{CH}_{3}\right), 22.8\left(\mathrm{CH}_{3}\right), 29.8,29.9(\mathrm{C}-8, \mathrm{C}-9), 33.1\left(\mathrm{CH}\left(\mathrm{CH}_{3}\right)_{2}\right)$, 36.1 (C-10), 42.5 (C-7), 46.4, 46.5, 46.6 (C-1, C-5, C-6), 50.9 (C-2), 60.3 (C-4). - MS (EI): $m / z$ (\%) 264.2 (4) $\left[\mathrm{M}^{+}\right]$, 249.1 (3), 221.1 (100), 73.0 (12). - HRMS (EI): calcd. for $\mathrm{C}_{16} \mathrm{H}_{28} \mathrm{OSi} 264.1909$; found $264.1899\left[\mathrm{M}^{+}\right]$.

rac-3-Methyl-2,7a-bis(trimethylsilyl)-2,3,3a,4,7,7a-hexahydro-1H-4,7-methanoinden-1-one (13)

$R_{f}=0.65$. - FT-IR (ATR): $v=2952$ (s), $2896(\mathrm{~m})$, 1685 (vs), 1454 (m), 1407 (m), 1374 (w), 1245 (vs), 1205 (m), 1183 (m), 1149 (vs), 1070 (m), 1043 (m), 915 (m), 832 (s), 735 (s), 688 (s), 618 (s) $\mathrm{cm}^{-1}$. ${ }^{1} \mathrm{H}$ NMR $\left(500.15 \mathrm{MHz},\left(\mathrm{CD}_{3}\right)_{2} \mathrm{CO}\right): \delta=0.02\left(\mathrm{~s}, 9 \mathrm{H}, 7 \mathrm{a}-\mathrm{Si}\left(\mathrm{CH}_{3}\right)_{3}\right)$, $0.12\left(\mathrm{~s}, 9 \mathrm{H}, 2-\mathrm{Si}\left(\mathrm{CH}_{3}\right)_{3}\right), 1.15\left(\mathrm{~d}, J=6.8 \mathrm{~Hz}, 3 \mathrm{H}, \mathrm{CH}_{3}\right)$, $1.32\left(\mathrm{td}, J=1.7 \mathrm{~Hz}, J=8.3 \mathrm{~Hz}, 1 \mathrm{H}, 10-\mathrm{H}_{\mathrm{a}}\right), 1.48(\mathrm{~d}$, $J=9.7 \mathrm{~Hz}, 1 \mathrm{H}, 4-\mathrm{H}), 1.49-1.51\left(\mathrm{~m}, 1 \mathrm{H}, 10-\mathrm{H}_{\mathrm{b}}\right), 1.83$ $(\mathrm{m}, 1 \mathrm{H}, 5-\mathrm{H}), 2.55(\mathrm{t}, J=4.0 \mathrm{~Hz}, 1 \mathrm{H}, 6-\mathrm{H}), 3.03(\mathrm{~m}$, $1 \mathrm{H}, 1-\mathrm{H}), 3.07(\mathrm{~m}, 1 \mathrm{H}, 7-\mathrm{H}), 6.08(\mathrm{dd}, J=3.0 \mathrm{~Hz}, J=$ $5.5 \mathrm{~Hz}, 1 \mathrm{H}, 8-\mathrm{H}), 6.20(\mathrm{dd}, J=2.7 \mathrm{~Hz}, J=5.5 \mathrm{~Hz}, 1 \mathrm{H}$, 9-H). $-{ }^{13} \mathrm{C} \mathrm{NMR}\left(125.76 \mathrm{MHz},\left(\mathrm{CD}_{3}\right)_{2} \mathrm{CO}\right): \delta=-0.9(7 \mathrm{a}-$ $\left.\mathrm{Si}\left(\mathrm{CH}_{3}\right)_{3}\right), 0.0\left(2-\mathrm{Si}\left(\mathrm{CH}_{3}\right)_{3}\right), 25.4\left(\mathrm{CH}_{3}\right), 35.9(\mathrm{C}-5), 49.1$ (C-7), 50.0 (C-1), 52.4 (C-10), 54.6 (C-4), 56.3 (C-6), 136.2

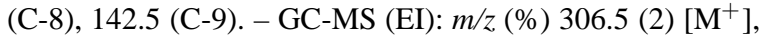
291.3 (2), 240.4 (35), 233.3 (30), 147.2 (9), 133.1 (23), 73.0 (100), 66.1 (20). - HRMS (EI): calcd. for $\mathrm{C}_{17} \mathrm{H}_{30} \mathrm{OSi}_{2}$ 306.1835; found $306.1839\left[\mathrm{M}^{+}\right]$.

\section{General procedure for the desilylation}

The respective desilylation agent ( 1.5 equiv.) was added to a solution of the appropriate enone $\mathbf{2}, \mathbf{3 a}, \mathbf{8 a}, \mathbf{b}, \mathbf{1 0}, \mathbf{1 2}$, or 13 (1.0 equiv.) in the respective solvent given in Tables 1,2 , and the reaction mixture stirred at the given temperature and times (Tables 1,2) (tlc control). Then the reaction mixture was filtered through $\mathrm{SiO}_{2}$ with $\mathrm{Et}_{2} \mathrm{O}$ and the filtrate was concentrated. The conversion was estimated by GC. The novel products were purified by chromatography on $\mathrm{SiO}_{2}$ with pentane/Et ${ }_{2} \mathrm{O}(15: 1)$ for $\mathbf{1}$ or pentane/Et ${ }_{2} \mathrm{O}(10: 1)$ for $\mathbf{1 1}$. Physical and spectroscopic data of compounds 11a and 14 were in accordance with those in the literature $[13,15]$.

rac-3-Methyl-3,3a,4,6a-tetrahydropentalen-1(2H)-one (1a)

$R_{f}=0.21 .-$ FT-IR (ATR): $v=3061(\mathrm{~m}), 2953(\mathrm{~s})$, 2923 (s), 2870 (m), 2848 (m), 1733 (vs), 1457 (m), 1410 (m), $1378(\mathrm{~m}), 1344(\mathrm{~m}), 1286(\mathrm{w}), 1213(\mathrm{~s}), 1172$ (s), $909(\mathrm{~m})$, $772(\mathrm{~m}) \mathrm{cm}^{-1} .-{ }^{1} \mathrm{H}$ NMR $\left(500.15 \mathrm{MHz}, \mathrm{CDCl}_{3}\right): \delta=1.13$ $\left(\mathrm{d}, J=6.7 \mathrm{~Hz}, 3 \mathrm{H}, \mathrm{CH}_{3}\right), 1.72-1.83(\mathrm{~m}, 1 \mathrm{H}, 3-\mathrm{H}), 1.95$ (ddd, $J=1.4 \mathrm{~Hz}, J=11.1 \mathrm{~Hz}, J=17.0 \mathrm{~Hz}, 1 \mathrm{H}, 2-\mathrm{H}_{\mathrm{a}}$ ), $2.24\left(\mathrm{dd}, J=7.0 \mathrm{~Hz}, J=17.0 \mathrm{~Hz}, 1 \mathrm{H}, 2-\mathrm{H}_{\mathrm{b}}\right), 2.26-2.32$ 
$\left(\mathrm{m}, 1 \mathrm{H}, 4-\mathrm{H}_{\mathrm{a}}\right), 2.48(\mathrm{dq}, J=1.3 \mathrm{~Hz}, J=6.7 \mathrm{~Hz}, 1 \mathrm{H}, 3 \mathrm{a}-\mathrm{H})$, 2.64 (dddd, $J=2.7 \mathrm{~Hz}, J=5.1 \mathrm{~Hz}, J=8.0 \mathrm{~Hz}, J=16.9 \mathrm{~Hz}$, $\left.1 \mathrm{H}, 4-\mathrm{H}_{\mathrm{b}}\right), 3.30-3.35(\mathrm{~m}, 1 \mathrm{H}, 6 \mathrm{a}-\mathrm{H}), 5.54(\mathrm{td}, J=2.4 \mathrm{~Hz}$, $J=7.6 \mathrm{~Hz}, 1 \mathrm{H}, 5-\mathrm{H}), 5.75(\mathrm{dt}, J=2.4 \mathrm{~Hz}, J=7.6 \mathrm{~Hz}, 1 \mathrm{H}$, 6-H). $-{ }^{13} \mathrm{C} \mathrm{NMR}\left(125.76 \mathrm{MHz}, \mathrm{CDCl}_{3}\right): \delta=20.8\left(\mathrm{CH}_{3}\right)$, 38.5 (C-3), 40.1 (C-4), 47.9 (C-2), 48.0 (C-3a), 62.3 (C-6a), 129.3 (C-5), 133.6 (C-6), 217.2 (C-2). - GC-MS (EI): $\mathrm{m} / \mathrm{z}$ (\%) 136.1 (56) $\left[\mathrm{M}^{+}\right], 107.1$ (10), 94.1 (44), 79.0 (32), 66.0 (100). - HRMS (EI): calcd. for $\mathrm{C}_{9} \mathrm{H}_{12} \mathrm{O}$ 136.0888; found $136.0888\left[\mathrm{M}^{+}\right]$.

rac-3-Butyl-3,3a,4,6a-tetrahydropentalen-1(2H)-one (1b)

$R_{f}=0.26$. FT-IR (ATR): $v=3062(\mathrm{~m}), 2956(\mathrm{~s})$, 2920 (s), 2872 (m), 2852 (s), 1733 (vs), 1465 (m), 1410 (m), 1379 (w), 1344 (w), 1287 (w), 1230 (m), 1213 (m), 1168 (s), 1116 (m), 910 (s), 732 (s), 689 (vs) $\mathrm{cm}^{-1} \cdot-{ }^{1} \mathrm{H}$ NMR $\left(500.15 \mathrm{MHz}, \mathrm{CDCl}_{3}\right): \delta=0.91\left(\mathrm{t}, J=6.9 \mathrm{~Hz}, 3 \mathrm{H}, 4^{\prime}-\right.$ H), $1.24-1.39\left(\mathrm{~m}, 5 \mathrm{H}, 1^{\prime}-\mathrm{H}_{\mathrm{a}}, 2^{\prime}-\mathrm{H}, 3^{\prime}-\mathrm{H}\right), 1.56-1.64(\mathrm{~m}$, $\left.1 \mathrm{H}, 1^{\prime}-\mathrm{H}_{\mathrm{b}}\right), 1.70-1.79(\mathrm{~m}, 1 \mathrm{H}, 3-\mathrm{H}), 1.94$ (ddd, $J=1.5 \mathrm{~Hz}$, $\left.J=11.0 \mathrm{~Hz}, J=17.6 \mathrm{~Hz}, 1 \mathrm{H}, 2-\mathrm{H}_{\mathrm{a}}\right), 2.26-2.32(\mathrm{~m}, 1 \mathrm{H}$, $\left.4-\mathrm{H}_{\mathrm{a}}\right), 2.39\left(\mathrm{dd}, J=7.2 \mathrm{~Hz}, J=17.4 \mathrm{~Hz}, 1 \mathrm{H}, 2-\mathrm{H}_{\mathrm{b}}\right), 2.52$ $(\mathrm{dq}, J=0.9 \mathrm{~Hz}, J=8.0 \mathrm{~Hz}, 1 \mathrm{H}, 3 \mathrm{a}-\mathrm{H}), 2.64-2.72(\mathrm{~m}$, $\left.1 \mathrm{H}, 4-\mathrm{H}_{\mathrm{b}}\right), 3.37-3.42(\mathrm{~m}, 1 \mathrm{H}, 6 \mathrm{a}-\mathrm{H}), 5.61(\mathrm{td}, J=2.3 \mathrm{~Hz}$, $J=7.7 \mathrm{~Hz}, 1 \mathrm{H}, 5-\mathrm{H}) 5.76(\mathrm{dt}, J=2.4 \mathrm{~Hz}, J=5.0 \mathrm{~Hz}$, $1 \mathrm{H}, 6-\mathrm{H}) .-{ }^{13} \mathrm{C}$ NMR $\left(125.76 \mathrm{MHz}, \mathrm{CDCl}_{3}\right): \delta=13.9$ (C-4'), 22.8 (C-3'), 30.2 (C-2'), 35.4 (C-1'), 39.4 (C-4), 42.1 (C-3), 44.7, 44.9 (C-2, C-3a), 60.7 (C-6a), 127.3 (C-5), 132.1 (C-6), 217.9 (C-2). - GC-MS (EI): $m / z(\%) 178.3(36)\left[\mathrm{M}^{+}\right]$, 149.2 (7), 136.2 (28), 121.2 (25), 111.1 (30), 107.1 (15), 92.1 (44), 79.1 (37), 66.1 (100), 55.1 (26). - HRMS (EI): calcd. for $\mathrm{C}_{12} \mathrm{H}_{18} \mathrm{O}$ 178.1358; found $178.1358\left[\mathrm{M}^{+}\right]$.

rac-3-Phenyl-3,3a,4,6a-tetrahydropentalen-1(2H)-one (1c)

$R_{f}=0.11 .-$ FT-IR (ATR): $v=3062(\mathrm{~m}), 3028(\mathrm{~m})$, 2899 (s), 2847 (s), 1732 (vs), 1601 (m), 1494 (s), 1453 (s), 1407 (m), 1342 (w), 1261 (m), 1211 (m), 1186 (s), 1168 (s), $1114(\mathrm{~m}), 909(\mathrm{~s}), 832(\mathrm{~m}), 759(\mathrm{~s}), 686(\mathrm{vs}) \mathrm{cm}^{-1}$. ${ }^{1} \mathrm{H}$ NMR $\left(500.15 \mathrm{MHz}, \mathrm{CDCl}_{3}\right): \delta=2.32-2.38(\mathrm{~m}, 1 \mathrm{H}$, $\left.4-\mathrm{H}_{\mathrm{a}}\right), 2.47\left(\mathrm{dd}, J=7.3 \mathrm{~Hz}, J=17.1 \mathrm{~Hz}, 1 \mathrm{H}, 2-\mathrm{H}_{\mathrm{a}}\right), 2.58-$ $2.63\left(\mathrm{~m}, 2 \mathrm{H}, 2-\mathrm{H}_{\mathrm{b}}, 4-\mathrm{H}_{\mathrm{b}}\right), 2.87-2.94(\mathrm{~m}, 1 \mathrm{H}, 3-\mathrm{H}), 3.05$ $(\mathrm{q}, J=8.4 \mathrm{~Hz}, 1 \mathrm{H}, 3 \mathrm{a}-\mathrm{H}), 3.50-3.55(\mathrm{~m}, 1 \mathrm{H}, 6 \mathrm{a}-\mathrm{H}), 5.63$ $(\mathrm{dt}, J=2.4 \mathrm{~Hz}, J=7.4 \mathrm{~Hz}, 1 \mathrm{H}, 5-\mathrm{H}), 5.82(\mathrm{dt}, J=2.6 \mathrm{~Hz}$, $J=5.2 \mathrm{~Hz}, 1 \mathrm{H}, 6-\mathrm{H}) .-{ }^{13} \mathrm{C} \mathrm{NMR}\left(125.76 \mathrm{MHz}, \mathrm{CDCl}_{3}\right)$ : $\delta=39.8$ (C-4), 48.2, 48.4 (C-2, C-3a), 49.5 (C-3), 62.5 (C-6a), 128.4, 129.1, 129.3, 130.4, 133.4 (C-5,0 C-6, C-2', C-3', C-4', C-5', C-6'), 145.2 (C-4'), 216.2 (C-2). - GCMS (EI): $m / z(\%) 198.2(42)\left[\mathrm{M}^{+}\right], 155.1$ (20), 133.1 (18), 115.1 (12), 104.1 (100), 91.0 (23), 66.1 (59). - HRMS (EI): calcd. for $\mathrm{C}_{14} \mathrm{H}_{14} \mathrm{O}$ 198.1045; found $198.1037\left[\mathrm{M}^{+}\right]$.

rac-3-Butyloctahydro-1H-4,7-methanoinden-1-one (11b)

$R_{f}=0.35 .-$ FT-IR (ATR): $v=2952$ (s), 2919 (s), 2871 (s), 1731 (vs), 1457 (m), $1411(\mathrm{w}), 1378$ (w), $1311(\mathrm{w})$,
1297 (w), 1250 (m), 1198 (s), 1183 (s), 1168 (s), 1119 (w), 1097 (w), 968 (w), 951 (w), 917 (w) $\mathrm{cm}^{-1} \cdot-{ }^{1} \mathrm{H}$ NMR $\left(500.15 \mathrm{MHz}, \mathrm{CDCl}_{3}\right): \delta=0.91(\mathrm{t}, J=7.0 \mathrm{~Hz}, 3 \mathrm{H}, 4$ '$\mathrm{H}), 1.02-1.08(\mathrm{~m}, 2 \mathrm{H}, 10-\mathrm{H}), 1.11-1.21\left(\mathrm{~m}, 2 \mathrm{H}, 8-\mathrm{H}_{\mathrm{a}}\right.$, 9- $\left.\mathrm{H}_{\mathrm{a}}\right), 1.30-1.39$ (m, $\left.4 \mathrm{H}, 2^{\prime}-\mathrm{H}, 3^{\prime}-\mathrm{H}\right), 1.44-1.66$ (m, $5 \mathrm{H}$, $\left.1^{\prime}-\mathrm{H}, 5-\mathrm{H}, 8-\mathrm{H}_{\mathrm{b}}, 9-\mathrm{H}_{\mathrm{b}}\right), 1.86(\mathrm{dd}, J=6.7 \mathrm{~Hz}, J=8.9 \mathrm{~Hz}$, $1 \mathrm{H}, 6-\mathrm{H}), 1.95$ (ddd, $J=1.6 \mathrm{~Hz}, J=11.4 \mathrm{~Hz}, J=17.2 \mathrm{~Hz}$, $\left.1 \mathrm{H}, 4-\mathrm{H}_{\mathrm{a}}\right), 2.12-2.19$ (m, $\left.3 \mathrm{H}, 2-\mathrm{H}, 4-\mathrm{H}_{\mathrm{b}}, 7-\mathrm{H}\right), 2.41-2.43$ (m, $1 \mathrm{H}, 1-\mathrm{H}) .-{ }^{13} \mathrm{C} \mathrm{NMR}\left(125.76 \mathrm{MHz}, \mathrm{CDCl}_{3}\right): \delta=15.3$ (C-4'), 24.5 (C-3'), 29.7, 29.8 (C-8, C-9), 32.1 (C-2'), 36.0 (C-10), 38.9 (C-1'), 40.8 (C-1), 41.0 (C-5), 44.1 (C-7), 47.1 (C-4), 52.6 (C-6), 57.5 (C-2). - MS (EI): $\mathrm{m} / z$ (\%) 206.2 (41) $\left[\mathrm{M}^{+}\right], 177.1$ (8), 149.1 (100), 139.0 (30), 121.1 (19), 111.0 (59), 95.0 (20), 79.0 (21), 66.0 (43), 55.0 (14). - HRMS (EI): calcd. for $\mathrm{C}_{14} \mathrm{H}_{22} \mathrm{O} 206.1671$; found $206.1671\left[\mathrm{M}^{+}\right]$.

rac-3-Phenyloctahydro-1H-4,7-methanoinden-1-one (11c)

$R_{f}=0.32 .-$ FT-IR (ATR): $v=3061(\mathrm{w}), 3027(\mathrm{~m})$, 2949 (s), 2871 (s), 1732 (vs), 1693 (s), 1601 (m), 1494 (s), 1452 (s), 1410 (m), 1384 (m), 1297 (m), 1268 (m), 1198 (s), 1180 (s), 1117 (m), 1092 (m), $944(\mathrm{w}), 914(\mathrm{~m}) \mathrm{cm}^{-1}$. ${ }^{1} \mathrm{H}$ NMR $\left(500.15 \mathrm{MHz}, \mathrm{CDCl}_{3}\right): \delta=1.09-1.15(\mathrm{~m}, 2 \mathrm{H}$, 9- $\left.\mathrm{H}_{\mathrm{a}}, 10-\mathrm{H}_{\mathrm{a}}\right), 1.15-1.20\left(\mathrm{~m}, 1 \mathrm{H}, 9-\mathrm{H}_{\mathrm{b}}\right), 1.23-1.27(\mathrm{~m}, 1 \mathrm{H}$, $\left.10-\mathrm{H}_{\mathrm{b}}\right), 1.48-1.56(\mathrm{~m}, 2 \mathrm{H}, 8-\mathrm{H}), 2.26-2.33$ (m, $2 \mathrm{H}, 6-\mathrm{H}$, 7-H), 2.36-2.42 (m, $\left.2 \mathrm{H}, 2-\mathrm{H}, 4-\mathrm{H}_{\mathrm{a}}\right), 2.51-2.54(\mathrm{~m}, 1 \mathrm{H}$, $1-\mathrm{H}), 2.58$ (ddd, $J=1.7 \mathrm{~Hz}, J=12.6 \mathrm{~Hz}, J=16.7 \mathrm{~Hz}, 1 \mathrm{H}$, $\left.4-\mathrm{H}_{\mathrm{b}}\right), 2.87(\mathrm{td}, J=7.6 \mathrm{~Hz}, J=12.5 \mathrm{~Hz}, 1 \mathrm{H}, 5-\mathrm{H}), 7.20-$ 7.39 (m, 5 H, 2'-H, 3'-H, 4'-H, 5'-H, 6'-H). - ${ }^{13} \mathrm{C}$ NMR $\left(125.76 \mathrm{MHz}, \mathrm{CDCl}_{3}\right): \delta=29.6,29.7(\mathrm{C}-8, \mathrm{C}-9), 35.9$ (C-10), 40.8 (C-1), 43.4 (C-6), 46.8 (C-5), 48.5 (C-4), 54.8 (C-7), 57.7 (C-2), 128.1 (C-4'), 129.0 (C-3', C-5'), 130.4 (C-2', C-6'), 147.4 (C-1'), 219.0 (C-3). - GC-MS (EI): $\mathrm{m} / \mathrm{z}$ (\%) $226(68)\left[\mathrm{M}^{+}\right], 130$ (13), 115 (8), 104 (100), 91 (14), 78 (28), 66 (42), 51 (16). - HRMS (EI): calcd. for $\mathrm{C}_{16} \mathrm{H}_{18} \mathrm{O}$ 226.1358; found $226.1354\left[\mathrm{M}^{+}\right]$.

rac-3-Isopropyloctahydro-1H-4,7-methanoinden-1-one (11d)

$R_{f}=0.35 .-$ FT-IR (ATR): $v=2953(\mathrm{~s}), 2871$ (s), 1731 (vs), 1652 (w), 1601 (m), 1469 (m), 1410 (w), 1385 (m), 1368 (m), 1201 (m), 1172 (m), 1095 (w), 864 (w) $\mathrm{cm}^{-1} .-{ }^{1} \mathrm{H}$ NMR $\left(300.13 \mathrm{MHz}, \mathrm{CDCl}_{3}\right): \delta=0.90(\mathrm{~d}$, $\left.J=6.6 \mathrm{~Hz}, 3 \mathrm{H}, \mathrm{CH}_{3}\right), 1.00\left(\mathrm{~d}, J=6.6 \mathrm{~Hz}, 3 \mathrm{H}, \mathrm{CH}_{3}\right)$, $1.02-1.05(\mathrm{~m}, 2 \mathrm{H}, 10-\mathrm{H}), 1.10-1.51(\mathrm{~m}, 5 \mathrm{H}, 5-\mathrm{H}, 8-\mathrm{H}$, 9-H), 1.59 (sept, $\left.J=6.6 \mathrm{~Hz}, 2 \mathrm{H}, \mathrm{CH}\left(\mathrm{CH}_{3}\right)_{2}\right), 1.90-2.20$ (m, 4 H, 2-H, 4-H, 6-H, 7-H), 2.40-2.42 (m, 1 H, 1-H). ${ }^{13} \mathrm{C}$ NMR $\left(75.47 \mathrm{MHz}, \mathrm{CDCl}_{3}\right): \delta=22.1\left(\mathrm{CH}_{3}\right), 22.2$ $\left(\mathrm{CH}_{3}\right), 29.6,29.7(\mathrm{C}-8, \mathrm{C}-9), 35.9(\mathrm{C}-10), 36.5\left(\mathrm{CH}\left(\mathrm{CH}_{3}\right)_{2}\right)$, 40.9 (C-1), 44.9 (C-7), 45.4 (C-4), 48.2 (C-5), 50.6 (C-6), 57.9 (C-2), 220.1 (C-3). - MS (EI): $\mathrm{m} / z$ (\%) 192.2 (63) $\left[\mathrm{M}^{+}\right.$], 149.1 (100), 121.1 (22), 97.1 (14), 79.1 (12), 66.1 (19), 41.0 (6). - HRMS (EI): calcd. for $\mathrm{C}_{13} \mathrm{H}_{20} \mathrm{O}$ 192.1514; found $192.1518\left[\mathrm{M}^{+}\right]$. 


\section{Crystal structure determination of $\mathbf{1 2}$}

Suitable single crystals were obtained by crystallization from $\mathrm{Et}_{2} \mathrm{O}$. Data were collected on a Siemens P4 diffractometer with graphite-monochromated $\mathrm{Cu}-\mathrm{K}_{\alpha}$ radiation $(\lambda=1.54178 \AA)$ at $293 \mathrm{~K}$. The structures were solved by direct methods and refined against $F^{2}$ for all observed reflections. Crystal data: $\mathrm{C}_{16} \mathrm{H}_{26} \mathrm{OSi}_{2}, M=290.6$, orthorhombic, space group $P 2{ }_{1} 2_{1} 2_{1} ; a=6.6924(4)$, $b=14.4241(8), c=18.4173(14) \AA, \alpha=\beta=\gamma=90^{\circ}$; $V=1777.9(2) \AA^{3}, Z=4 ; D_{\mathrm{c}}=1.086 \mathrm{~g} \mathrm{~cm}^{-3}$. Data collection: crystal size $0.3 \times 0.25 \times 0.20 \mathrm{~mm}, 3177$ reflections in the range $\theta=3.89-67.48^{\circ}, 2726$ unique reflections. Structure refinement: 2726 reflection data $(I>2 \sigma(I))$, 173 parameters; the final $R$ indices were $R=0.0670$, $R \mathrm{w}=0.0898$; residual electron density between 0.344 and $-0.227 \mathrm{e}^{-3}$.

[1] a) L. V. R. Bonaga, M.E. Krafft, Tetrahedron 60, 9795 (2004); b) S. E. Gibson, A. Stevenazzi, Angew. Chem. 115, 1844 (2003); Angew. Chem. Int. Ed. 42, 1800 (2003); c) B. E. Hanson, Comments Inorg. Chem. 23, 289 (2002); d) K. M. Brummond, J. L. Kent, Tetrahedron 56, 3263 (2000); e) A. J. Fletcher, S.D.R. Christie, J. Chem. Soc. Perkin Trans. 11657 (2000); f) Y. K. Chung, Coord. Chem. Rev. 188, 297 (1999).

[2] Recent examples of alternative pentalenone synthesis: a) J. Yang, Y. O. Lang, L. A. Paquette, J. Am. Chem. Soc. 125, 1567 (2003); b) A. N. M. M. Rahman, R. Bishop, D.C. Craig, M. L. Scudder, Eur. J. Org. Chem. 72 (2003); c) A. Wrobleski, K. Sahasrabudhe, J. Aubé, J. Am. Chem. Soc. 124, 9974 (2002); d) H. Hashimoto, Y. Abe, Y. Mayuzumi, M. Karikomi, K. Seki, K. Haga, T. Uyehara, Tetrahedron Lett. 43, 265 (2002); e) J. Barluenga, S. Martinez, A. L. Suárez-Sobrino, M. Tomás, J. Am. Chem. Soc. 124, 5948 (2002); f) G. Mehta, K. Sreenivas, Tetrahedron Lett. 43, 3319 (2002); g) S. Kotha, R. Sivakumar, L. Damodharan, V. Pattabhi, Tetrahedron Lett. 43, 4523 (2002); h) L. A. Paquette, J. Yang, Y. O. Long, J. Am. Chem. Soc. 124, 6542 (2002); i) H.-P. Wu, R. Aumann, R. Fröhlich, E. Wegelius, Organometallics 20, 2183 (2001); j) G. Mehta, K. Srinivas, Tetrahedron Lett. 42, 2855 (2001); k) D. M. Hodgson, I.D. Cameron, M. Christlieb, R. Green, G.P. Lee, L. A. Robinson, J. Chem. Soc. Perkin Trans. 12161 (2001); 1) E. Piers, A. Orellana, Synthesis 2138 (2001); m) R. Muto, K. Ogasawara, Tetrahedron Lett. 42, 4143 (2001).

[3] I. U. Khand, P.L. Pauson, M. J. A. Habib, J. Chem. Res. (S) 348 (1978).

[4] N. E. Schore, B. E. La Belle, M. J. Knudsen, H. Hope, X.-J. Xu, J. Organomet. Chem. 272, 435 (1984).
Crystallographic data for the structure have been deposited with the Cambridge Crystallographic Data Centre, CCDC-290468. Copies of the data can be obtained free of charge on application to The Director, CCDC, 12 Union Road, Cambridge, CB2 1EZ, UK (Fax: int.code+(1223)336033; e-mail for inquiry: fileserv@ @cdc.cam.ac.uk).

\section{Acknowledgements}

Generous financial support by the Deutsche Forschungsgemeinschaft, the Fonds der Chemischen Industrie and the Ministerium für Wissenschaft, Forschung und Kunst des Landes Baden-Württemberg is gratefully acknowledged. A. Becheanu thanks the Deutsche Akademische Austauschdienst for a DAAD award and T. Bell thanks the European Community for an ERASMUS/SOKRATES fellowship.

[5] K.E. B. Parkes, G. Pattenden, J. Chem. Soc. Perkin Trans. 11119 (1988).

[6] a) X. Verdaguer, A. Lledó, C. Lopez-Mosquera, M. A. Maestro, M. A. Pericas, A. Riera, J. Org. Chem. 69, 8053 (2004); b) J. Castro, A. Moyano, M. A. Pericas, A. Riera, A. Alvarez-Larena, J. F. Piniella, J. Am. Chem. Soc. 122, 7944 (2000); c) P. Magnus, L. M. Principe, M. J. Slater, J. Org. Chem. 52, 1483 (1987); d) E. Carceller, V. Centellas, A. Moyano, M. A. Pericas, F. Serratosa, Tetrahedron Lett. 26, 2475 (1985); e) C. Exon, P. Magnus, J. Am. Chem. Soc. 105, 2477 (1983).

[7] X. Verdaguer, A. Moyano, M. A. Pericas, A. Riera, M. A. Maestro, J. Mahia, J. Am. Chem. Soc. 122, 10242 (2000).

[8] a) W. J. Kerr, D. M. Lindsay, M. McLaughlin, P. L. Pauson, Chem. Commun. 1467 (2000); b) M. Iqbal, Y. Li, P. Evans, Tetrahedron 60, 2531 (2004).

[9] J. L. Loebach, D. M. Bennett, R. L. Danheiser, J. Am. Chem. Soc. 120, 9690 (1998).

[10] R. Ducray, N. Cramer, M. A. Ciufolini, Tetrahedron Lett. 42, 9175 (2001).

[11] I. U. Khand, G. R. Knox, P.L. Pauson, W.E. Watts, M. I. Foreman, J. Chem. Soc. Perkin Trans. 1977 (1973).

[12] J.-B. Wiel, F. Rouessac, J. Chem. Soc. Chem. Commun. 446 (1976).

[13] a) T. Sakan, K. Abe, Tetrahedron Lett. 2471 (1968); b) P.P. M. A. Dols, M. M. H. Verstappen, A. J. H. Klunder, B. Zwanenburg, Tetrahedron 49, 11353 (1993).

[14] M. Iqbal, P. Evans, A. Lledó, X. Verdaguer, M. A. Pericas, A. Riera, C. Loeffler, A. K. Sinha, M. J. Mueller, ChemBioChem 6, 276 (2005).

[15] L. N. Ivanova, T. A. Severina, G. A. Kogan, V.F. Kucherov, Izv. Akad. Nauk SSSR, Ser. Khim. 1438 (1963); Chem. Abstr. 59, 13838b (1963). 